\title{
Lithium Ions Batteries Electrodes Materials, Design, Outlook and Future Perspectives
}

\author{
Xiangrui Li \\ Year 2 undergraduate, The Chinese University of Hong Kong (Shenzhen), China
}

\begin{abstract}
With the rapid growth of lithium-ion batteries (LIBs) industry and market, it is important to develop LIBs with higher capacities, stability and lifespan. However, challenges like low conductivity, solid electrolyte interface formation and electrode cracking now are in the way, which are closely related to electrode deficiency. Hence, to obtain better performance, the main orientation is to improve electrode materials' morphological structure. Better porosity, stability and conductivity of anode and cathode materials tangibly improve the performance of LIBs including coulombic efficiency, safety and cyclic lifespan. This review paper will combine current challenges, commonly used and emerging electrode materials together with battery testing methods and their market trend to provide a big picture about LIBs.
\end{abstract}

\section{Introduction}

Due to the rapid consumption of fossil fuels for modern industry and transportation together with the rapid evolution in electrical storage, it is in a pressing need to find other energy sources to replace the vacancy of fossil fuel to keep the operation of industry and catch up the market trend. The environmental concerns of greenhouse gas emission boost the development of clean energy like solar, hydroelectric and wind etc. Therefore, LIBs that are rechargeable with high energy density trait are becoming a significant direction for future energy management.

Different kinds of batteries will have different structures. Take tesla cell as an example. As shown in figure 1, this lithium-ion battery (LIB) is composed of copper, aluminium as anode and cathode electrodes. It takes graphite as anode material and lithium nickel cobalt aluminium oxide as cathode with a separator and electrolyte between them. This structure is a standard form of LIBs and its Swiss-roll like structure maximize its amount of active material for more energy[1].

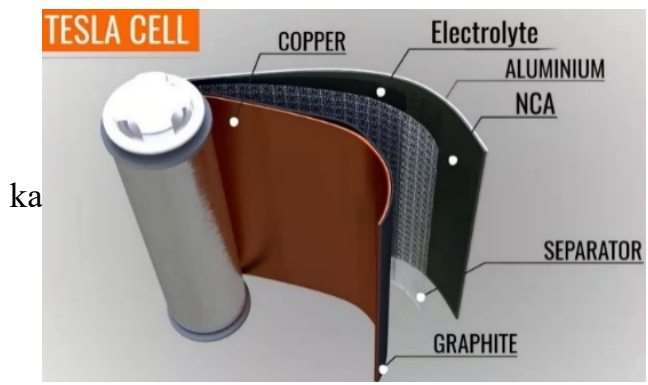

Figure 1 Lithium-ion batteries (LIBs) simplified structure

Although nowadays portable electronic devices are important consumer of LIBs, the expectation for higher capacity and large-scale equipment like electrical vehicle (EV) and grid storage also requires for high power and capacity. Recent LIBs are still insufficient in specific capacity/volumetric capacity and power density. Despite that the electric vehicles have already been commercialized at present; challenges are still evident that the modern electric vehicle nowadays can only travel for about $60-450 \mathrm{~km}$ according to the NEDC test cycle [2]. For the battery configuration, it takes up as large as 0.1$0.6 \mathrm{~m}^{3}$ [3]. Besides, for limousine-class electric vehicle which has a travel range more than 300 kilometres, the weight of battery carried can even exceed $500 \mathrm{~kg}$ [3] (figure 2). Therefore, here comes a stringent need in assembling the next generation of LIBs with superior charge and high power that suitable for electric vehicle industry, electric appliances, and space applications [4].

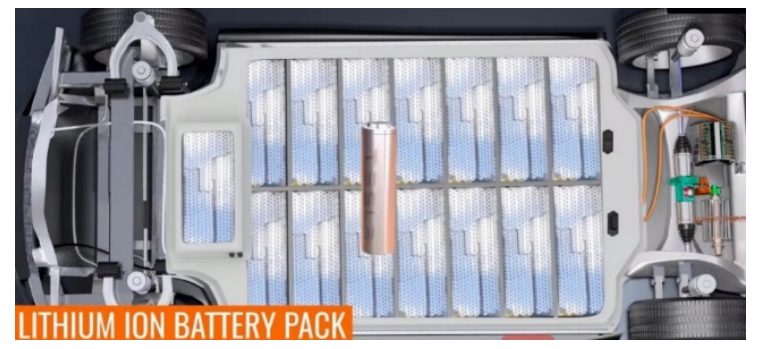

Figure 2 Lithium-ion battery pack in electrical vehicle [1]

However, recent LIBs still need to be improved to match the growing demand of capacity and life cycle. The energy density of LIBs mainly depends on the mechanism of anode and cathode materials. Nanotechnology, which is an accessible science in enhancing the performance of LIBs by combining with electrode materials. Nanomaterials refers to materials that smaller than $100 \mathrm{~nm}$ in three dimensions and cover a large span from organic 
to inorganic, nonmetal to metal. These nanomaterials can be customized into specified structure for different usage and have wide applications in electrode development.

\section{Challenges Associated with Li-ion Batteries}

\subsection{Low Conductivity and Thermal Runaway}

For battery, it is common that there will be capacity loss due to the low conductivity of the materials. Thermal runway is also an electrochemical problem inevitable caused by the internal impedance of the battery. Thermal runaway is a major concern for LIBs application [2], and this problem is commonly occurred in the lithium transition metal oxide, for example, lithium cobalt oxide has the lowest thermal stability of any commercial cathode material [37]. This problem will cause the energy runoff in the form of heat when operating. Heat dissipated from the LIBs is mainly generated by activation (interfacial kinetics), concentration (related to the transportation of species) and ohmic losses (resistance) during the electron movement [4]. To reduce the low conductivity and thermal runaway, electrode and electrolyte materials with low impedance should be exploited. Commonly used method is to incorporate the carbon or highly conductive agents at the external surface or the inner surface of electrode [5]. All these issues related to the electrochemical property of materials and it's important to figure out their chemical behaviours to control the thermal runaway.

\subsection{Morphological and Structural/Phase Changes}

Morphological structure is a vital characteristic of electrode materials and it is a key concern in the LIBs since it relates to the process of $\mathrm{Li}$ insertion and extraction, which can primarily affect the battery performance. Moreover, morphological variations will accumulate isolated products that lead to the electrical contact loss that obliterates the cell operation [4]. Not only morphological variation, materials will also display crystal structure and phase deviation that affect the battery capacity and lifespan [4]. Change in crystal structure will cause defective conductivity and inactive kinetics for $\mathrm{Li}+$ insertion and extraction process, incurring poor capacities and lifespan [6]. Therefore, to solve this problem, scientists involve nanoscience in to refine materials' nanostructure that can partially diminish the morphological deviations by reinforced phase conversion kinetics [4].

\subsection{Volume Changes and Thickness of Solid Electrolyte Interface Film}

Volume change happens during $\mathrm{Li}+$ alloying/dealloying process because $\mathrm{Li}+$ will apply strains to the electrodes. Solid Electrolyte Interface (SEI) is a layered substance that usually forms on the surface of anode and cathode due to the electrochemical reduction of the electrolyte and usually occurs during the initial few charge-discharge cycles of LIBs [32]. The formation of the SEI is connected with the electrochemical properties of the electrolyte and the electrode materials and will affect the performance of battery including its capacity, safety and life cycle [2, 4, 32]. During the charging/discharging process, the movement of particles (ions and electrons) leads to the redox reaction between Li-ions, electrons and electrolyte (mostly lithium salts like LiPF6 and organic solution) (Figure 3a) and form the SEI layer. As for unsustainable SEI, it cannot sustain stress caused by the volume change and easily breakdown. This breakdown will further lead to the exposure of electrode and SEI formation (Figure $3 \mathrm{~b}$ ), but it can be predicted by the morphological and volumetric variations for proactive control [4]. Simultaneously, it can also serve as a protective coating of the electrode that allows lithium ions to pass for intercalation but not electrons [42]. Therefore, it can somehow prevent the further degradation of the electrolyte. Under this condition, it is important to control the thickness of SEI to maximize the battery performance. However, up to now, there are no preferable mechanisms to restrain the SEI layer compositions, grain size, thickness and spatial distribution [4]. Hence, morphology of electrode materials affects the formation of SEI film predominantly. Customized electrode morphology and encasing the appearance with inactive materials like carbon coatings will improve our control over SEI film [4].

\subsection{Cracking and Fracture of Particles and Electrodes and Electron/ion Transport}

Cracking of particles and electrodes commonly occurs during the lithium intercalation/ extraction, resulting the further corrosion of electrode from electrolyte through these cracks. During the intercalation and deintercalation process, the frequent egress and ingress of $\mathrm{Li}+$ will apply strains on electrode materials and ultimately cause the cracks. Generally, traditional intercalation-type electrode materials undertake subtle volume variations smaller than $10 \%$. On the contrary, novel electrode materials with high capacity suffer greater volume variations because of large lithium intake [4]. The large variation in the volume during lithium intercalation/extraction process is considered as a crucial issue in restricting the performances and applications of high-capacity electrodes.

Alloy materials are generally characterized with high capacity due to large lithium intake and therefore will undertake great volume variation, for example, $420 \%$ for $\mathrm{Si}, 300 \%$ for $\mathrm{P}$, and $260 \%$ for Ge and $\mathrm{Sn}$ [4]. These drastic volume changes lead to the electrodes, active materials potential, and the cycle life degradation [4]. To solve the problems of cracking and facture, commonly used method is to coat the electrodes with materials that nonreactive to the electrolyte to prevent the direct contact between electrode and electrolyte, and then restrain the erosion from electrolyte. These materials should be permeable for 
lithium ions but hinder the electron movement to restrict the formation of SEI.

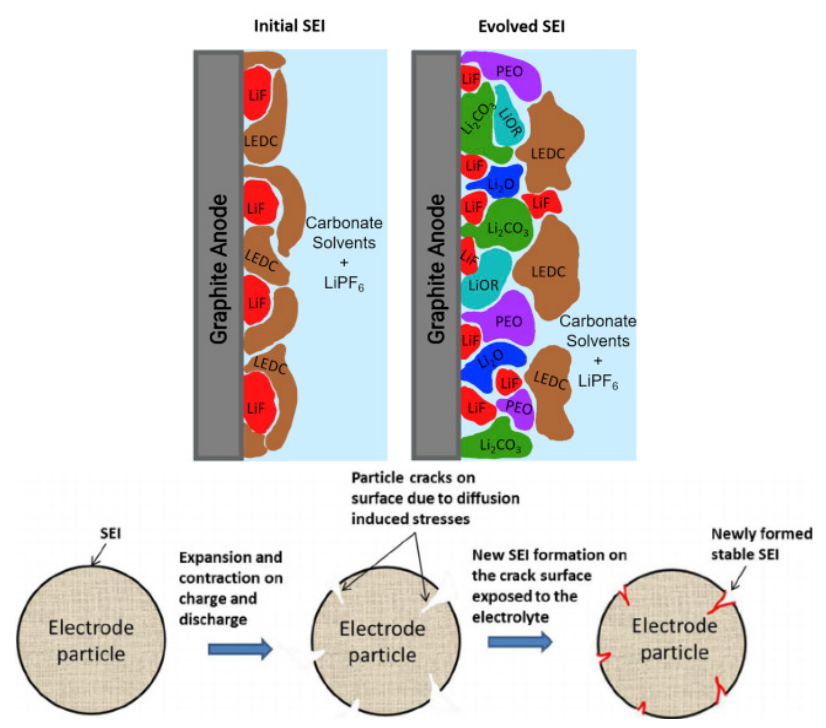

Figure 3 (a) SEI composition and distribution [7]; (b) Electrode fracture and SEI formation process [8]

\section{Anode Materials}

\subsection{Carbon-Based Electroactive Materials}

Carbon-based materials include carbon nanotubes (CNTs), graphene, fullerenes and other materials that composed of carbon atoms arranging in different morphological structure (Figure 4). Commonly used materials for anode electrode are CNTs, graphene who have excellent electron conductivity, stability and transportation ability for lithium intercalation [4, 43]. Besides, carbon-based materials with attractive benefits of relatively low cost, moderate energy density, power density and electrochemical stabilities, who are suitable for LIB anode materials $[2,4]$.

Conventionally, graphite is one of the most commonly used 2D carbon-based anode materials. It is composed of sp2 carbon atoms with honeycomb-like structure [4]. And it derivative - graphene, has drawn great attention due to its impressive properties and extensive applications in many fields, including physical, chemical, and biological [9]. Its special latticed single layer morphological structure contributes to excellent electron conductivity, mechanical strength, well mobility and great specific surface, making it possible to be a competitive candidate in LIB applications [10].

As for the CNTs, it is a universal 1D nanomaterials used in fabricating porous body and combining with other anodic materials to improve electrode performance due to its excellent electronic conductivity, thermal, mechanical stabilities and good transportation rate [2, 4, 43]. CNTs have various structures. It can be roughly categorized into single-walled carbon nanotubes (SWCNTs) and multiwalled nanotubes (MWCNTs) [4]. It also has different grid structures including armchair, zigzag and chiral, where armchair is conductive, zigzag is semiconductive and chiral is insulative.

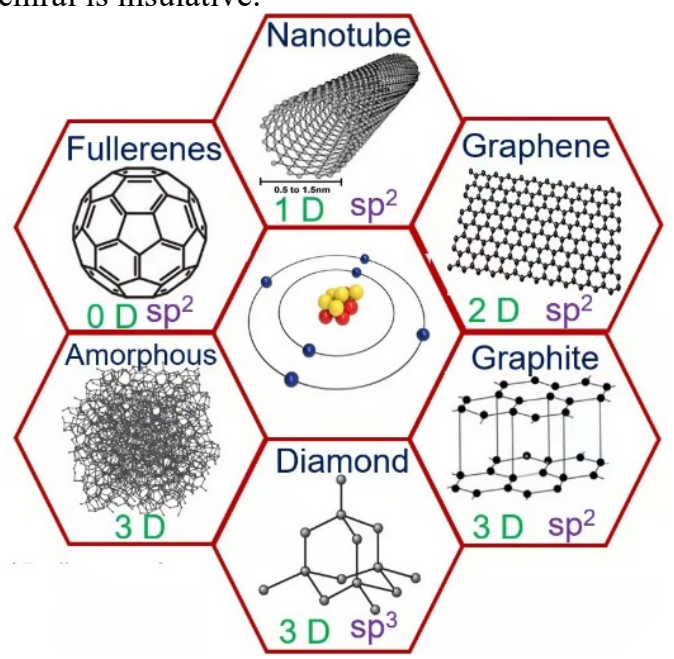

Figure 4 Structures of different kinds of carbon-based materials [12]

\subsection{Titanium-Based Oxides Materials}

$\mathrm{Li}_{4} \mathrm{Ti}_{5} \mathrm{O}_{12}$ is one of the memebr that has been successfully commercialized because it excellently combines the factors of outstanding thermal stability [13], relatively high volumetric capacity, and long-lasting lifespan, inspite of flaws like inferior cell voltage, high cost and low capacity ( $\left.175 \mathrm{~m} \mathrm{Ah} \mathrm{g}^{-1} \& 600 \mathrm{~m} \mathrm{Ah} \mathrm{cm}^{-3}\right)$ [2]. It was also considered "zero strain" under high potential lithiation/delithiation because the phase change is tiny $(0.2 \%)$ in volume [2]. Therefore, this slight volume change can increase the stability of both electrode and SEI film. Furthermore, LTO can offer high potential above 1V that can avoid the formation and growth of SEI film. The high potential also prevents the $\mathrm{Li}$ dendrite formation which will cause capacity loss and bad safety [2].

Due to the property of the titanium-based oxide, the gassing reaction on the surface is inevitable between organic electrolyte and LTO [38], which will cause the capacity loss. This process can be suppressed by coating carbon materials but the involving of carbon materials also lead to problems of catalyzing electrolyte decomposition $[14,15]$. But in general, LTO anode can last for tens of thousands cycles and become a competitive material in high power application [39].

\subsection{Alloy materials (Si, Gr, Metal oxides, Metal Phosphides)}

Alloy materials are characterized with high capacity. Germanium, silicon, silicon oxide and tin oxide are anodic materials that have high capacity taking place in lithium reaction correlated to alloy/de-alloy phenomenon [4]. Their specific capacity can range from $783 \mathrm{~mA} \mathrm{~h} \mathrm{~g}^{-1}$ for tin oxide to $4211 \mathrm{~mA} \mathrm{~h} \mathrm{~g}^{-1}$ for silicon [16-18]. However, this trait also leads to the electrode expansion and further cause short lifespan. There are two solutions regarding to this problem: narrowing the particles size to nanoscale and manufacturing compositions with the combination of active lithium and inert materials [4]. With nanoscale 
particles, the strain given to the electrode will be decrease and protect the electrode. For the second method, active/inactive lithium material is more receptive by this composite because it can be regarded as a buffer between the inert materials and active lithium [19], which makes intercalation easier.

Germanium is a potential anode material with relative high capacity (1623 $\mathrm{mA} \mathrm{h} \mathrm{g}^{-1}$ ) compared to common anodes [4]. But it has drawbacks of higher cost and lower capacity in comparision with silicon, it is competitive in the fields of higher electronic conductivity that 104 times greater than silicon and narrow bandwidth potential $(0.67$ eV) [4]. Even so, the practical application of germanium is hampered due to its large volume variation (300\%) [4]. That's why nanoarchitectures are introduced to eliminate this kind of problem. Nanotubes, nanowires and nanoparticles [20,21,22] can conspicuously constraint the volume change more efficiently when compared to bulk and microstructure. These nanoarchitectures can work as a structural buffer to restrict the volume variation and can even reduce the formation of SEI layer [4].

Silicon is an emerging anodic material with extremely high capacity (e.g : $\mathrm{Li}_{22} \mathrm{Si}_{5}, 4200 \mathrm{~mA} \mathrm{~h} \mathrm{~g}^{-1}$ ) [4]. It is also abundant on the earth with relatively low price compared to lithium. $\mathrm{Si}$ is an outstanding candidate due to its relatively low delithiation potential, high gravimetric/ volumetric capacity, chemical stability, reserves, and nontoxicity [2]. Like other alloy materials, it will suffer great variation in volume. To solve this problem, silicon can be combined to MXene that form interspaces to orderly receive $\mathrm{Li}$-ions and prevent expansion (figure 5).

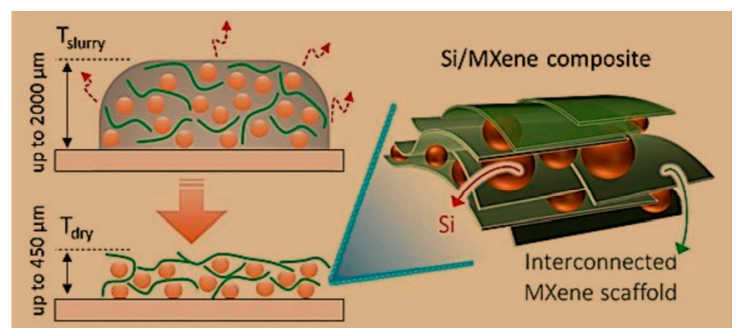

Figure 5 Slurry-casting process of Si/MXene combination and its structure [4]

Silicon oxide has capacity over $1600 \mathrm{~mA} \mathrm{~h} \mathrm{~g}{ }^{-1}$, lower volume charge and simultaneously low activation of energy [4]. SiO also represents a dominating disadvantage: it is not thermally stable at various temperatures [4] and will be converted to $\mathrm{SiO}_{2}$ at high temperature ambience, which will damage the capacity and lifetime of the battery. The involvement of oxygen atom will lead to the formation of Si-Li alloy and also the lithium silicates [4] during the discharge process, and this can be described in the following equation:

$$
\mathrm{xLi}+\mathrm{SiO} \leftrightarrow \mathrm{Li}_{\mathrm{x}}-\mathrm{ySi} \mathrm{z}_{\mathrm{z}}+\mathrm{Li}_{\mathrm{x}} \mathrm{SiO}_{\mathrm{z}}
$$

\section{Cathode Materials}

\subsection{Lithium Cobalt Oxide (LiCoO2)}

Lithium cobalt oxide is a widely used cathodic material in LIBs. $\mathrm{LiCoO}_{2}$ (LCO) can provide layer structure for lithiation and the lithium cations lie in between two layers of composition of cobalt and oxygen atoms like sandwich. LCO is a fantasting cathode material with high specific capacity (274 mA h g $\left.\mathrm{m}^{-1}\right)$, high volumetric capacity (1363

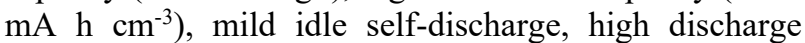
voltage, and good cycling performance $[40,41]$. The major limitations of it are low thermal stability, and fast capacity fade at high current or deep cycling [2]. This low thermal stability is reflected in high temperature performance, which will cause exothermic oxygen release and lead to flame [2].

\subsection{Lithium Nickel Oxide (LiNiO2)}

Lithium nickel oxide is characterized by high capacity which is widely used in portable devices and it can offer specific capacity for about $200 \mathrm{~m} \mathrm{Ah} \mathrm{g}^{-1}$ [4]. Compared with LCO, it has similar structure to allow the lithium-ion intercalation but harder to produce because the trivalent nickel is unstable in high temperature environment [4]. And LNO's cyclic stability is poor, which is ascribed to the cation mixing [44]. Also, LNO is unstable at high temperature environment because the +3 nickel is unstable at that situation [4]. Therefore, there is method called doping to improve its drawbacks by involving other particles [4].

\subsection{Lithium Manganese Oxide (LiMnO2)}

Lithium manganese oxide has many different structures and $\mathrm{LiMnO}_{2}$ is one of them that has layered structure for lithium intercalation and suitable for cathode material. $\mathrm{LiMnO}_{2}$ (LMO) is a commonly used cathode in primary cell which can also be used in rechargeable cell thanks to its low cost, envrionmental implication, and cost-effective alternative to LCO and LNO [4]. But its cyclic performance is not satisfied because its layered structure is not stable during the extraction and Mn will run off during the charge-discharge process [2], providing a bad cyclic stability.

\subsection{Combination}

The combination cathode refers to the combination of cobalt, nickel and manganese in the form of $\mathrm{Li}\left[\mathrm{Ni}_{1-\mathrm{x}-\mathrm{y}} \mathrm{Co}_{\mathrm{x}}\right.$ $\left.\mathrm{Al}_{\mathrm{y}}\right] \mathrm{O}_{2}$ to acquire the advantages of above transition metals, where nickel can provide high specific capacity, cobalt can offer enhanced rate capability and manganese can enhance structural integrity with its layered structure together with better safety in battery $[4,23]$. Therefore, the proportion of each element in the cathode determine the performance of the final product. Recently, there are researches about the high nickel LIB, like NCM631, NCM 811 (numbers after stands for the ratio of different components). Although it has some outstanding point like 
high capacity, but high nickel content simultaneously means that the cobalt and manganese contents will be lower, which will cause the overall structural instability and serious capacity fading [25], also with safety issues [4]. High nickel content will also form spinel at the surface, the inactive phase Fm (-) $3 \mathrm{~m}$ and the fatigue capacity that damage its performance [4] [26].

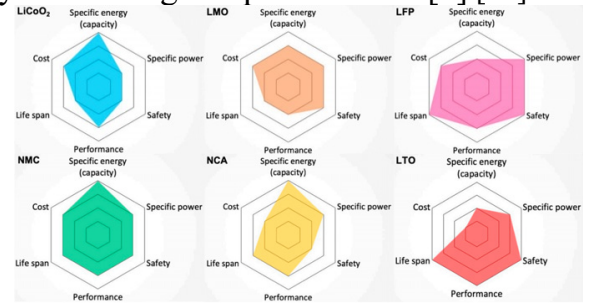

Figure 6 Comparisons of different LIBs in the perspectives of specific energy, power, safety, performance, lifespan, and cost.

\subsection{Iron Fluoride}

Generally, the mono electron diffusion involved during the intercalation process limited the capacity of LIBs [27]. Iron trifluoride has become an incaparably important material because of its eminent features of low cost, nontoxity and satisfactory thermal stabilities [28]. And the emerging material $\mathrm{FeF}_{3}$ can provide eminent capacity, energy density and great voltage output because of its high electronegativity from both iron and fluoride [4]. The manufacturing process of $\mathrm{FeF}_{3}$ so far mostly involves in solution which means that the employment of HF is unavoidable [28]. The HF has strong corrosivity, toxicity so that it is dangerous for mass production and hinder its commercialization.

\subsection{Vanadium Oxide}

Among aforementioned cathode materials, vanadium pentoxide $\left(\mathrm{V}_{2} \mathrm{O}_{5}\right)$ and other vanadium-based oxides have their attractive features including enhanced specific capacity, low cost, facile synthesis and abundance which stand out and comparable for LIB cathode material [4]. Vanadium pentoxide has orthorhombic structure and interconnect each molecular by sharing the oxygen atom, then forms layered structure and each layer can be restrained by an der Waals forces. This layered structure offers good capacity for $\mathrm{Li}^{+}$intercalation and extraction, resulting good synthesized capacity value up to $294 \mathrm{~mA} \mathrm{~h}$ $\mathrm{g}^{-1}$ [29]. Moreover, vanadium is relative common and has lower price compared to lithium (Figure 6).

However, the practical use of $\mathrm{V}_{2} \mathrm{O}_{5}$ is restricted according to its low $\mathrm{Li}+$ diffusion rate $\left(10^{-12}-10^{-13} \mathrm{~cm}^{2} \mathrm{~s}^{-1}\right)$, restricted conductivity $\left(10^{-2}-10^{-3} \mathrm{~S} \mathrm{~cm}^{-1}\right)$ and unstable structure. All these factors will slow the ions and electron transportation and lead to poor cyclic stability and overall performance [30] [31].

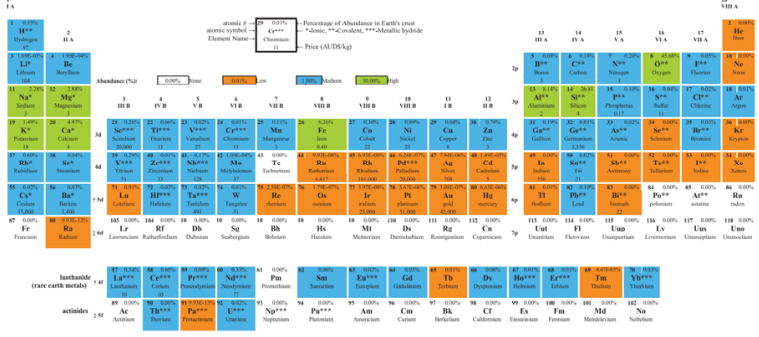

Figure 7 Price and abundance of elements in periodic table

\section{Li-ion battery Testing}

\subsection{Galvanostatic charge-discharge cycling behaviour}

The galvanostatic charging and discharging behaviors are important factors to obtain the voltage, charging or discharging behaviors and columbic efficiency (CE). The galvanostatic charge-discharge curve (GCD) is a commonly used chart for analyzing battery cycling behavior. As shown in figure $8 \mathrm{a}$, the GCD curve always consist of an ascending line and a descending line which represent the charging and discharging process relatively. From the curve, the voltage behavior can be observed according to the y-axis. Furthermore, the charging capacity of the battery can be obtained if we calculate the integral before the summit point. Likewise, the discharging capacity can be obtained and then the CE can be calculated by the formula:

$$
C E=\frac{Q_{c}}{Q_{d}}=\frac{A_{b}}{A_{a}}
$$

where $\mathrm{Q}_{\mathrm{c}}, \mathrm{Q}_{\mathrm{d}}$ represents the charge capacity and discharge capacity, $A_{b}$ represents area before summit and $A_{a}$ stands for area after summits. Generally, the symmetric curve means the $100 \% \mathrm{CE}$ but a concave curve connected to a convex curve represents $\mathrm{CE}<100 \%$. The GCD curve also can present charge/discharge curves separately with state of charge (SoC) $\mathrm{x}$-axis (Figure $8 \mathrm{~b}$ ) or specific capacity, all these depend on the requirement of experiment.

\subsection{Cyclic stability}

For all kinds of batteries, their performance will fluctuate due to its internal variation after serval cycles. Therefore, to test and improve battery stability, cyclic stability is an important indicator to be measured. Shown here in 8c, it represents the specific capacity and coulombic efficiency of different materials at the same time and it can also take volumetric capacity as y-axis as well. Generally, the capacity retention of the battery will decrease due to lithium loss and other factors and then tend to be stable. However, it can be observed that the coulombic efficiency will have a sharp increase at the beginning oppositely then become stable. The reason is that for a fresh battery, it takes time for SEI layer to form during the innitial several cycles[32]. From this diagram, capacity and CE variation and their steady state are generally important target that should be paid attention to. 


\subsection{Internal resistance}

Open circuit voltage is a primary characteristic of a battery. Open circuit voltage is the voltage between the positive and negative terminal of the battery when there is no discharging current or loads. The value of the open circuit voltage is determined by the redox reaction of the active materials and it can be directly measured by the voltameter or multimeter directly. For practical use, the battery cannot reach to open circuit voltage because of its internal resistance when current applied. The internal resistance of battery will take away some voltage. With the voltage take away by battery and current through it, its internal resistance can be sketchily calculated by

$$
R=\frac{V_{b}}{I_{b}}
$$

where $\mathrm{V}_{\mathrm{b}}$ represents voltage taken by internal resistance and $I_{b}$ represents the current through it. Hence, when fabricating the battery, the pursuit of low internal resistance is always important in reducing the thermal runaway and energy loss.

\subsection{Nyquist plots}

Nyquist plot is the diagram to obtain material's impedance. Firstly, it is important to know the concept of impedance. Impedance refers to the ability of the electrolyte that prevents the ions to move freely to the electrode and related to the ion diffusion rate. This resistance includes the real part resistance like a resistor and also imaginary part resistance always contributed by a capacitor. Generally, the real part is denoted as $\mathrm{R}$ and the imaginary part is presented in the imaginary number $\mathrm{j}$ $(\mathrm{j}=$ sqrt $(-1))$. And Electrical impedance spectroscopy (EIS) is the method that used to research on the impedance of the battery and analysis its diffusion coefficient. The Nyquist plot is the direct demonstration of EIS in different frequencies.

Like figure.1, the Nyquist plot is set up in a coordinate system of $x$-axis (real part) and y-axis (imaginary part) with function $\mathrm{Z}(\mathrm{w})$, where $\mathrm{Z}$ represents the impedance and $w$ represents the frequency. The plots start with high frequency of a semicircle to low frequency of a sloping line, where semicircle represents the reaction process and sloping line refers to diffusion process respectively [45]. And the first intersection point refers to the solution resistance and the second intersection point represents charge transfer resistance [45]. The impedance caused by diffusion process is also called Warburg impedance which is related to diffusion rate. Therefore, data from the Nyquist plot can be used to compute the diffusion coefficient.

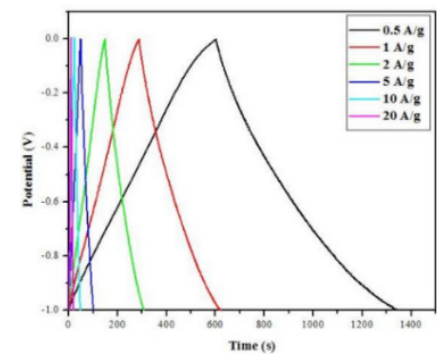

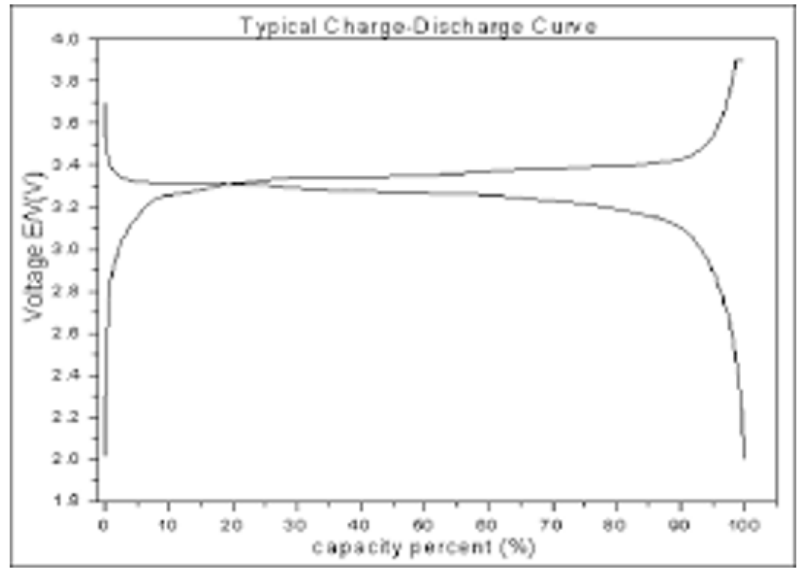
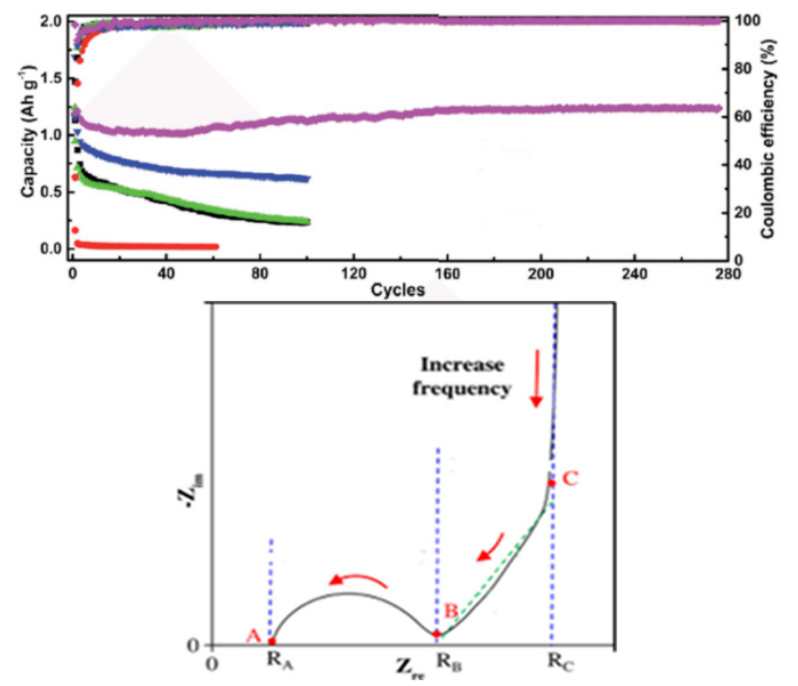

Figure 8 (a) Galvanostatic charge-discharge (GCD) curve with different specific current [6]; (b) Charge-discharge curve [33] (c)charge-discharge curves that represent the capacity and coulombic efficiency variation of different materials (represented by different colors) [34] (d) Nyquist plots. The red pointer points from high frequency to low frequency [35]

\section{Market Trends}

Nowadays, there exist a large number of companies dealing with nanomaterials and battery fabrication because of the growing demand from fields like electronic devices, transportation and grid storage.

As table 4 shows, portable devices including mobile phones, tablets, laptops and other electronic devices had become the primary LIBs market which constitute about $80 \%$ of the share at the time of 2015 and is still increasing steadily. The cosumers demand varied over years, for example, from the table, it can be seen that the annual LIB demand for laptops (PC) is relatively stable at about 10 GWh, while more portable devices are being preferred and gowing firmly. The LIB demand for cell phones and tablets is growing at an average speed of $10 \%$ annually. From 2015 to 2020 the average annual growth rate of portable devices is $9 \%$, and $10 \%$ from 2025 in 2030 .

For the transportation, LIBs are mostly used in electrical vehicle (EV), hybrid electric vehicle (HEV) and plug-in hybrid electric vehicle (PHEV). The market trend is exceeding the portable electronic field because of the growing of new energy vehicle industry. In 2025, the 
estimated demand for road transportation will reach to $137 \mathrm{GWh}$ and $245 \mathrm{GWh}$ in 2030 . The average annual growth rate from 2025 to 2030 is $16 \%$, which is considered to take the place of the leading position of portable devices.

Also, the emergence of energy storage is a latent consumer of LIBs in the future. LIBs can be utilized as storage units in power supply systems due to its high capacity and modularization trait for both grid-connected (centralized) and off-grid (distributed) [36]. Until 2015 the share of storage is still $0 \mathrm{GWh}$ but grow in a rapid rate in the following years, the estimated annual growth rate from to 2030 even reaches $40 \%$, which is a competitive market of LIBs in the future.

Table 1 Li-ion battery market, previous development and future prospect from 2010 to 2030 [36]

\begin{tabular}{llllll}
\hline & 2010 & 2015 & 2020 & 2025 & 2030 \\
\hline Cell phones & 6 & 11 & 17 & 28 & 44 \\
Tablets & 1 & 7 & 12 & 17 & 25 \\
PC & 12 & 9 & 9 & 9 & 11 \\
Portable electronics, other & 3 & 4 & 7 & 12 & 20 \\
Portable electronics, total & $\mathbf{2 1}$ & $\mathbf{3 1}$ & $\mathbf{4 5}$ & $\mathbf{6 6}$ & $\mathbf{1 0 0}$ \\
EV & 0 & 11 & 65 & 115 & 200 \\
PHEV & 0 & 2 & 8 & 13 & 25 \\
HEV & 0 & 0 & 2 & 7 & 15 \\
Road-transport, other & 0 & 0 & 1 & 2 & $\mathbf{5}$ \\
Road-transport, total & 0 & $\mathbf{1 3}$ & $\mathbf{7 6}$ & $\mathbf{1 3 7}$ & $\mathbf{2 4 5}$ \\
Storage in power supply & 0 & $\mathbf{0}$ & $\mathbf{2}$ & $\mathbf{1 0}$ & $\mathbf{3 0}$ \\
Other applications & $\mathbf{1}$ & $\mathbf{1}$ & $\mathbf{2}$ & $\mathbf{7}$ & $\mathbf{1 5}$ \\
Total & 22 & $\mathbf{4 5}$ & $\mathbf{1 2 5}$ & $\mathbf{2 2 0}$ & $\mathbf{3 9 0}$ \\
\hline
\end{tabular}

\section{Conclusion and Future Perspectives}

The aforementioned article has already presented a framework for LIBs current challenges, electrode materials, testing method and their market trend. LIB is an indispensable source in the future and nanomaterials are significant way to enhance its capability .

In the future, we are looking forward to batteries with better property (Figure 9), including better capacity, lower price, safety etc. The challenges nowadays are low conductivity, electrode fracture and SEI layer which are tightly related to the electrode physicochemical property and morphological structure. When considering about suitable electrode materials, basically their cost, conductivity, chemical property and morphological structure are always need to comprehensively considered. Not only materials themselves, the combination of different compositions can also auxiliary improve their abilities or shortcomings. Normally, the combination of nanomaterials including, graphene, CNT and other nanoparticles with electrode materials is a significant way in developing better electrode with excellent stability, conductivity and capacity.

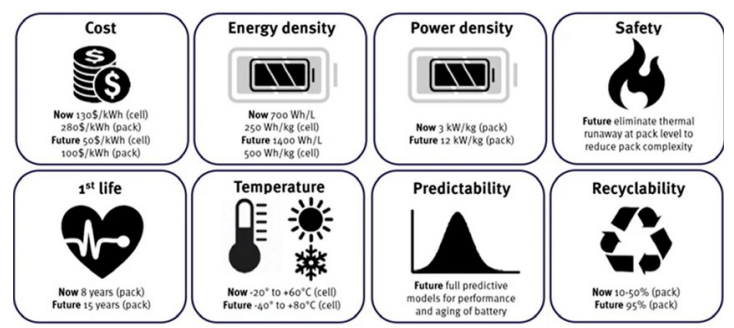

Figure 9 Significant improvement needed in the future [12].

Better capacity is always what we pursue, and researcher are trying hard to develop materials that can be apply extensively. For cathode materials, the combination electrodes especially nickel-rich cathode is developing rapidly with excellent capacity. Currently, a variety of NCM members like NCM333, NCM523 are already commercialized and the enhanced materials NCM811 and NCM622 are still under research and hopefully they can be deployed in the near future [4]. The testing method including GCD curve, Cyclic stability analysis and Nyquist plot are important in analyzing battery capacity, stability and conductivity in a visualized and quantified way. Hopefully, the battery market trend will definitely continue to rise, especially portable devices, electrical vehicle (EV)) and grid storage. Hence, it is important to match the steeply growing demand and various requirement of different equipment.

Even though electrode material is an important and potential factor to develop battery performance in the following years, there still other parts of battery like separator, electrolyte and internal structure can contribute to better LIBs which needs to be further studied.

\section{References}

1. Lithium-ion battery, how does it work? https://www.youtube.com/watch?v=VxMM4g2Sk8 U

2. Nitta N, Wu F, Lee J T, et al. Li-ion battery materials: present and future. Materials today, 2015, 18(5): 252264.

3. Thomas C.E. Fuel cell and battery electric vehicles compared. International Journal of Hydrogen Energy. 2009, 34 (15), 6005-6020.

4. Kim H J, Krishna $\mathrm{T} N$ V, Zeb K, et al. A comprehensive review of Li-ion battery materials and their recycling techniques. Electronics, 2020, 9(7): 1161.

5. Bai, P.; Bazant, M.Z. Charge transfer kinetics at the solid-solid interface in porous electrodes. Nat. Commun. 2014, 5, 1-7.

6. Son, M.Y.; Hong, Y.J.; Lee, J.K.; Chan Kang, Y. One-pot synthesis of $\mathrm{Fe} 2 \mathrm{O} 3$ yolk-shell particles with two, three, and four shells for application as an anode material in lithium-ion batteries. Nanoscale 2013, 5, 11592-11597.

7. Heiskanen S K, Kim J, Lucht B L. Generation and evolution of the solid electrolyte interphase of lithium-ion batteries. Joule, 2019, 3(10): 2322-2333.

8. Xu, J; Deshpande, Rutooj; Pan, J; Cheng, Y; Battaglia, V. Electrode Side Reactions, Capacity Loss and Mechanical Degradation in Lithium-Ion Batteries. Journal of The Electrochemical Society. 2015, 162. 2026-2035.

9. Stankovich, S.; Dikin, D.A.; Dommett, G.H.B. et al. Graphene-based composite materials. Nature 2006, 442, 282-286. 
10. Liang, M.; Zhi, L. Graphene-based electrode materials for rechargeable lithium batteries. J. Mater. Chem. 2009, 19, 5871-5878.

11. Brownson, D.A.C.; Kampouris, D.K.; Banks, C.E. An overview of graphene in energy production and storage applications. J. Power Sources 2011, 196, 4873-4885.

12. M. B. Bazbouz. Lithium-ion Battery Materials (Anode, Cathode and separator). 2021.

13. Doughty D H, Roth E P. A general discussion of Li ion battery safety. The Electrochemical Society Interface, 2012, 21(2): 37.

14. He Y B, Liu M, Huang Z D, et al. Effect of solid electrolyte interface (SEI) film on cyclic performance of Li4Ti5O12 anodes for $\mathrm{Li}$ ion batteries. Journal of Power Sources, 2013, 239: 269-276. 15. M.-S. Song, et al. J. Mater. Chem. 2014, A2(3), 631.

15. Reddy, A.L.M.; Gowda, S.R.; Shaijumon, M.M.; Ajayan, P.M. Hybrid nanostructures for energy storage applications. Adv. Mater. 2012, 24, 50455064.

16. Mekonnen, Y.; Sundararajan, A.; Sarwat, A.I. A review of cathode and anode materials for lithium-ion batteries. In Proceedings of the SoutheastCon 2016, Norfolk, VA, USA, 30 March-3 April 2016.

17. Kong, L.L.; Wang, L.; Ni, Z.C. et al. LithiumMagnesium Alloy as a Stable Anode for LithiumSulfur Battery. Adv. Funct. Mater. 2019, 29, 1808756.

18. Kasavajjula, U.; Wang, C.; Appleby, A.J. Nano- and bulk-silicon-based insertion anodes for lithium-ion secondary cells. J. Power Sources 2007, 29, 1808756.

19. Park, M.H.; Cho, Y.; Kim, K. et al. Germanium nanotubes prepared by using the Kirkendall effect as anodes for high-rate lithium batteries. Angew. Chem. Int. Ed. 2011, 50, 9647-9650.

20. Flynn, G.; Palaniappan, K.; Sheehan, M.; Kennedy, T.; Ryan, K.M. Solution synthesis of lead seeded germanium nanowires and branched nanowire networks and their application as Li-ion battery anodes. Nanotechnology 2017, 28(25): 255603.

21. Kim, C.H.; Im, H.S.; Cho, Y.J. et al. High-yield gasphase laser photolysis synthesis of germanium nanocrystals for high-performance photodetectors and lithium-ion batteries. J. Phys. Chem. C 2012, 50, 26190-26196.

22. Scrosati, B. History of lithium batteries. J. Solid State Electrochem. 2011, 15, 1623-1630.

23. Blomgren, G.E. The Development and Future of Lithium-Ion Batteries. J. Electrochem.Soc. 2017, 164, 1.

24. Hwang J, Do K, Ahn H. Highly conductive 3D structural carbon network-encapsulated Ni-rich LiNi0.8Co0.1Mn0.1O2 as depolarized and passivated cathode for lithium-ion batteries. Chemical Engineering Journal, 2021, 406: 126813

25. Is Li-Ion the Solution for the Electric Vehicle? https://batteryuniversity.com/learn/archive/is_li_ion _the_solution_for_the_electric_vehicle
26. Zheng, Q.; Yamada, Y.; Shang, R. et al. A cyclic phosphate-based battery electrolyte for high voltage and safe operation. Nat. Energy 2020, 5, 291-298.

27. Li, T.; Li, L.; Cao, Y.L. et al. Reversible threeelectron redox behaviors of FeF3 nanocrystals as high-capacity cathode-active materials for Li-Ion batteries. J. Phys. Chem. C 2010, 114, 3190-3195.

28. Chen, C.; Xu, X.; Chen, S. et al. The preparation and characterization of iron fluorides polymorphs $\mathrm{FeF} 3 \cdot 0.33 \mathrm{H} 2 \mathrm{O}$ and $\beta-\mathrm{FeF} 3 \cdot 3 \mathrm{H} 2 \mathrm{O}$ as cathode materials for lithium-ion batteries. Mater. Res. Bull. 2015, 64, 187-193.

29. Ma, Y.; Huang, A.; Zhou, H.; Ji, S.; Zhang, S.; Li, R.; Yao, H.; Cao, X.; Jin, P. Template-free formation of various $\mathrm{V} 2 \mathrm{O} 5$ hierarchical structures as cathode materials for lithium-ion batteries. J. Mater. Chem. 2017, 5, 6522-6531.

30. Lai Q, Sun Y, Wang T, et al. How to design hydrogen storage materials? Fundamentals, synthesis, and storage tanks. Advanced Sustainable Systems, 2019, 3(9): 1900043.

31. Smith A, Burns J, Trussler S, Dahn J. Precision measurements of the coulombic efficiency of lithium-ion batteries and of electrode materials for lithium-ion batteries. J Electrochem Soc 2010, 157(2): A196-202

32. Qaisar, S M; Dallet, D; Benjamin, S; et al. Power efficient analog to digital conversion for the Li-ion battery voltage monitoring and measurement. IEEE International Instrumentation and Measurement Technology Conference (I2MTC). IEEE, 2013: 1522-1525.

33. Shah, M S A S; Park, A R; Rauf, A et al. Highly interdigitated and porous architected ternary composite of $\mathrm{SnS} 2, \mathrm{gC} 3 \mathrm{~N} 4$, and reduced graphene oxide (rGO) as high-performance lithium-ion battery anodes. RSC Advances, 2017, 7(6): 3125-3135.

34. Mei, B., Munteshari, O., Lau, J., Dunn, B., \& Pilon, L. Physical Interpretations of Nyquist Plots for EDLC Electrodes and Devices. Journal of Physical Chemistry C, 2018, 122: 194-206.

35. Zubi G, Dufo-López R, Carvalho M, et al. The lithium-ion battery: State of the art and future perspectives. Renewable and Sustainable Energy Reviews, 2018, 89, 292-308.

36. Doughty D H, Roth E P. A general discussion of Li ion battery safety. The Electrochemical Society Interface, 2012, 21(2): 37.

37. He Y B, Li B, Liu M, et al. Gassing in Li4Ti5O12based batteries and its remedy. Scientific reports, 2012, 2(1): 1-9.

38. Zaghib K, Dontigny M, Guerfi A, et al. Safe and fastcharging Li-ion battery with long shelf life for power applications. Journal of power sources, 2011, 196(8): 3949-3954.

39. R. Yazami, et al. New Trends in Intercalation Compounds for Energy Storage and Conversion: 
Proceedings of the International Symposium. The Electrochemical Society, vol. 2003, 2003, p. 317.

40. Du Pasquier A, Plitz I, Menocal S, et al. A comparative study of Li-ion battery, supercapacitor and nonaqueous asymmetric hybrid devices for automotive applications. Journal of power sources, 2003, 115(1): 171-178.

41. Liu, N., Lu, Z., Zhao, J. et al. A pomegranate-inspired nanoscale design for large-volume-change lithium battery anodes. 2014, Nature Nanotech 9, 187-192.

42. Yu, Y.; Cui, C.; Qian, W. et al. Carbon nanotube production and application in energy storage. AsiaPac. J. Chem. Eng. 2013, 8, 234-245.

43. Ohzuku, T. Electrochemistry and Structural Chemistry of LiNiO2(R3m) for 4 Volt Secondary Lithium Cells. Electrochem. Soc. 1993, 147, 1862.

44. Intro to Electrochemical Impedance Spectroscopy (EIS) of Batteries. https: //www. youtube. com/watch?v=xaimI9w-egQ

45. Whittingham, M.S. Lithium batteries and cathode materials. Chem. Rev. 2004, 104, 4271-4302. 